\title{
Distributed Relative Clock Synchronization for Wireless Sensor Networks
}

\author{
Iman Shames * and Adrian N. Bishop ** \\ * ACCESS Linnaeus Centre, Royal Institute of Technology (KTH) \\ ** NICTA and the Australian National University (ANU)
}

\begin{abstract}
This paper considers an important problem in sensor networks, i.e. clock synchronization in wireless sensor networks with a focus on those scenarios where the inter-node time-of-arrival measurements are noisy. Initially, a simple convex, constraint based, optimization protocol for the problem of relative clock synchronization in wireless (sensor) networks is presented. Later, we provide a distributed discrete-time solution to the same problem and show exponential convergence. Then we provide a similar algorithm for achieving the same solution in continuous-time. Both the discrete-time algorithm and the continuous-time algorithm are distributed in that each node in the network requires very little information from its neighbours in the network. In the end, we provide a modification of the continuous time algorithm that achieves a finite-time convergence to the desired solution under some additional requirements.
\end{abstract}

\section{INTRODUCTION}

Wireless sensor networks provide a novel solution to a wide range of problems across a number of application areas. In many applications Cristian (1989); Sundararaman et al. (2005), individual sensor nodes are often required to attach so-called "where" and "when" information to the underlying sensed data.

The first question is addressed in those works that are concerned with the question of localization of sensor networks, e.g. convex optimization based localization algorithms (Doherty et al. (2001); Beck et al. (2008); Carter et al. (2006); Biswas et al. (2006); Biswas and Ye (2004); Ding et al. (2010)), algorithms using sum of squares relaxation (Nie (2009); Shames et al. $(2009 b, a)$ ), graph connectivity based algorithms (Shang et al. (2003); Lederer et al. (2009)), methods that use multidimensional scaling (Costa et al. (2006)), or other approaches described in Moore et al. (2004); Bruck et al. (2009); Bachrach and Taylor (2005).

The second question, which this paper is concerned with, is related to the more general problem of network synchronization, see Wang and Chen (2002); Barahona and Pecora (2002) and the references therein, and is referred to as the problem of clock synchronization. The problem of relative clock synchronization for wireless networks (or distributed networks more generally) is not new Cristian (1989); Sundararaman et al. (2005) and a number of protocols exist. We consider algorithms based on the time-of-arrival (TOA) stamps measured at individual sensors from a limited number of wireless signals transmitted by certain neighbour nodes in the network. The measurements at sensor $i$ typically take the form

$$
\widehat{t}_{i j}^{\bar{\kappa}}=t_{i j}^{\bar{\kappa}}+e_{i}^{\bar{\kappa}}=\tau_{i}^{\bar{\kappa}}+\frac{r_{i j}}{v}+e_{i}^{\bar{\kappa}}
$$

\footnotetext{
* Email: imansh@kth.se, adrian.bishop@anu.edu.au. This work was supported by NICTA, the Swedish Research Council (VR), and Knut and Alice Wallenberg Foundation. NICTA is funded by the Australian Government as represented by the Department of Broadband, Communications and the Digital Economy and the Australian Research Council through the ICT Centre of Excellence program.
}

for a signal, indexed by $\kappa$, transmitted from sensor $j$. The intersensor range is $r_{i j}$ and the speed of signal propogation is $v$. Here, $e_{i}^{\bar{\kappa}}$ represents the noise in the measurement. In general, we assume that $e_{i}^{\bar{\kappa}}$ and $e_{j}^{\bar{\kappa}}$ are independent. The time $\tau_{i}^{\bar{\kappa}}$ is the unknown time of transmission for message $\kappa$ measured in node $i$ 's internal time frame. We assume that node $j$ transmits

$$
\widehat{\tau}_{j}^{\bar{\kappa}}=\tau_{j}^{\bar{\kappa}}+n_{j}^{\bar{\kappa}}
$$

to node $i$ where $\tau_{j}^{\bar{\kappa}}$ is the time of signal transmission as measured in node $j$ 's time frame. We assume $n_{i}^{\bar{\kappa}}$ corresponds to the noise in this transmission.

Note that $t_{i j}^{\bar{\kappa}}=\tau_{i}^{\bar{\kappa}}+\frac{r_{i j}}{v}=\tau_{j}^{\bar{\kappa}}+\left(\tau_{i}^{\bar{\kappa}}-\tau_{j}^{\bar{\kappa}}\right)+\frac{r_{i j}}{v}$ and we can now introduce the following definition.

Definition 1. The relative clock bias between node $i$ and $j$ is given by $\beta_{i j}=\tau_{j}-\tau_{i}=-\beta_{j i}$. If node $i$ knows $\beta_{i j}$ for all nodes $j$ within node $i$ 's communication range then node $i$ is said to be synchronized. Moreover, if all nodes are synchronized then the network is said to be globally synchronized. Two nodes $i$ and $j$ within communication range are said to be adjacent.

We assume that events stamped in node $j$ 's internal clock as $\theta_{j}$ which are transmitted to an adjacent node $i$ are then transformed into node $i$ 's internal frame using $\theta_{i}=\theta_{j}-\beta_{i j}$.

The simplest approach for pair-wise clock synchronization is to compute the estimate

$$
\widetilde{\beta}_{i j}=\frac{\left(\widehat{t}_{j i}^{a}-\widehat{\tau}_{i}^{a}\right)-\left(\widehat{t}_{i j}^{b}-\widehat{\tau}_{j}^{b}\right)}{2}
$$

when $i$ and $j$ are adjacent. Node $i$ broadcasts a packet $a$ to node $j$ along with $\widehat{\tau}_{i}^{a}$. Node $j$ measures $\widehat{t}_{j i}^{a}$ and subsequently returns a packet $b$ to node $i$ along with $\widehat{t}_{j i}^{a}$ and $\widehat{\tau}_{j}^{b}$. Node $i$ then measures $\widehat{t}_{i j}^{b}$ and computes $\widetilde{\beta}_{i j}$. Given multiple measurements over time, and a Gaussian error assumption, then taking the average of $\widehat{\tau}_{j}^{i}$ results in the unconstrained maximum likelihood estimate (MLE) for $\widetilde{\beta}_{i j}$; see e.g. Sundararaman et al. (2005).

Assumption 1. The clock drift is neglible over short time intervals, e.g. those considered in this work. That is, the true 
relative bias $\beta_{i j}=\frac{1}{2}\left[\left(t_{j i}^{a}-\tau_{i}^{a}\right)-\left(t_{i j}^{b}-\tau_{j}^{b}\right)\right]$ is independent of the packet indices $a$ and $b$.

The solution to (3) is widely used and is a variant on the remote clock reading algorithm Cristian (1989). A wide range of distributed clock synchronization prototols are based on variants of this technique Sundararaman et al. (2005). A widely referenced technique known as the reference broadcast synchronization (RBS) protocol can be regarded as a simplified variant of the above approach Elson et al. (2002) where a single node is elected as a reference transmitter (at least within defined clusters) and the time difference between time stamps receieved at adjacent nodes amounts to a bias estimate. Other techniques such as the Timing-sync Protocol for Sensor Networks (TPSN) Ganeriwal et al. (2003), the Flooding-Time Synchronization Protocol (FTSP) Maroti et al. (2004), the Routing Integrated Time Synchronization protocol (RITS) Sallai et al. (2006) and others also use a variant of the above solution with various modifications, e.g. the broadast technique and toplogy, compensation for drift etc. For brevity, we point to the survey Sundararaman et al. (2005) for details.

Alternatively, a number of algorithms based on the idea of distributed consensus (developed in the control community Shames et al. (2009a)) have recently been introduced; see e.g. Schenato and Gamba (2007); Xiong and Kishore (2008); Bolognani et al. (2009). Many of these techniques have the advantage of being truly distributed. However, such protocols typically neglect the addition of noisy measurements and are often based on the notion of asymptotic convergence, i.e. they require continual execution, and none of them take advantage of the structure of the network to improve their performance. However, some of these techniques Sommer and Wattenhofer (2009); Bolognani et al. (2009) inherently solve the problem in the face of clock drift, albeit under ideal (noiseless) sensor conditions.

Additional, and more recent, detailed studies are provided in Simeone and Spagnolini (2007); Freris and Kumar (2007); Solis et al. (2006); Giridhar and Kumar (2006); Freris et al. (2009) where convergence and mathematically rigorous results concerning performance are provided. In particular, we refer to Freris and Kumar (2007). The work of Barooah and Hespanha (2005) is also related and discusses the general problem of distributed estimation using only relative measurements. The clock synchronization problem considered here is one such instance of a general estimation problem with relative measurements.

Our approach is based on the relative communication protocol and equation (3). We explicitly stated that the solution (3) is unconstrained as it naively neglects the constraints imposed on the relative clock biases by the existence of network cycles. For example, consider a simple network of three sensor nodes $\{i, j, k\}$ with a triangular network topology. Now it follows from simple algebra that

$$
\beta_{i j}+\beta_{j k}+\beta_{k i}=0
$$

and thus any solution to (3) must satisfy such a constraint. We are unaware of any similar algorithm in the literature which explicitly considers such constraints.

This paper extends the author's previous work Shames and Bishop (2010) on relative clock synchronization. In Shames and Bishop (2010) a simple centralized solution to the problem of clock synchronization for wireless sensor networks was introduced based on constraint opimization. That approach explicitly considered the constraints imposed on the solution by the network topology.

In this paper, after outlining some preliminary results, we extend Shames and Bishop (2010) and introduce a number of distributed mechanisms for solving the stated constrained optimization algorithm. Firstly, we provide a discrete-time solution that is guaranteed to converge to the optimal constrained linear least-squares solution with an exponential speed. Then we provide a similar algorithm for achieving the same solution in continuous-time. Both the discrete-time algorithm and the continuous-time algorithm are distributed in that each node in the network requires very little information from its neighbours in the network.

Finally, we provide an algorithm that achieves a finite-time convergence to the desired solution. This algorithm requires that each node in the network have access to knowledge concerning the network topology and in particular certain kinds of network cycles.

\section{CONSTRAINT-BASED CLOCK SYNCHRONIZATION}

We start this section by formally presenting the problem that we address in this paper.

Problem 1. Consider the network $\mathcal{N}$, with underlying graph $\mathcal{G}(\mathcal{V}, \mathcal{E})$, where $\mathcal{V}=\{i\}_{i=1}^{N}$ with $|\mathcal{V}|=N$, is its vertex set and $\mathcal{E}$ is its edge set. Moreover the undirected edge $\{i, j\} \in \mathcal{E}$ if and only if nodes $i$ and $j$ are adjacent in the network, in other words one can calculate a $\widetilde{\beta}_{i j}$ as in (3). The answers to the following questions are sought.

(1) In the absence of noise, under what conditions can one find $\beta_{i j}$ for any pair of nodes $i$ and $j$ in the network?

(2) In the presence of noise, how can one improve the calculated values of $\widetilde{\beta}_{i j}$ obtained from (3)?

It is common to say two nodes $i$ and $j$ are adjacent if they are in close physical proximity to one another, i.e. $\{i, j\} \in \mathcal{E}$ iff the range $r_{i j}$ is less than some threshold $d_{t}$. We do not restrict ourselves to this case however.

In the following theorem we answer the first question posed in Problem 1.

Theorem 1. All the relative clock biases in the network, $\beta_{i j}$, $\forall i, j \in \mathcal{V}$, can be calculated if and only if graph $\mathcal{G}$ is connected.

Now we address the second question posed in Problem 1 by proposing a method which takes a similar approach to the one proposed in Piovan et al. (2008) to improve the relative clock biases obtained from solving (3) in the presence of noise. Note that for every cycle in the network, we can enforce a constraint along the lines of (4). If $\mathcal{G}$ is a tree, then there are no redundant cyclic constraints and we cannot reduce the effect of noise on the bias estimates beyond the solution (3).

Before continuing further, we have the following definitions.

Definition 2. Define the directed edge set $\mathcal{E}_{d}$ as

$$
\left.\mathcal{E}_{d}=\{(i, j) \mid\{i, j\} \in \mathcal{E}, \& i<j)\right\},
$$

furthermore, let $\mathcal{G}_{d}$ denote the directed graph (digraph) with vertex set $\mathcal{V}_{d}$ and edge set $\mathcal{E}_{d}$. 
Definition 3 (Path and cycle). Let $\mathcal{G}$ be either a directed or undirected non-empty graph. A path is a non-empty graph $P=\left(V_{P}, E_{P}\right) \subset \mathcal{G}$ of the form $V_{P}=\{i\}_{i=1}^{k}$ and $E_{p}=\left\{\left(j_{i}, j_{i+1}\right)\right\}_{i=1}^{k-1}$, where $\left\{j_{1}, \cdots, j_{k}\right\}$ is a permutation of $\{1, \cdots, k\}$. The vertices $j_{2}, \cdots, j_{k-1}$ are the inner vertices of $P$. Furthermore, every sequence of edges that form a closed path in $\mathcal{G}$ and do not visit the same node twice, except the start/end node, is called cycle and it is denoted by $\Lambda$.

The direction of a cycle is the order in which the nodes are visited. We let $L(\mathcal{G})$ denote the set of all cycles of $\mathcal{G}$, and $|\Lambda|$ denote the number of edges in the cycle $\Lambda$.

Definition 4 (Cycle vector). For $\Lambda \in L\left(\mathcal{G}_{d}\right)$, the cycle vector is the vector $1_{\Lambda} \in\{-1,0,+1\}^{|\mathcal{E}|}, \Lambda \subset \mathbb{R}^{|\mathcal{E}|}$ whose $i$-th entry is +1 if the $i$-th edge belongs to $\Lambda$ and its orientation is consistent with the orientation of $\Lambda,-1$ if the $i$-th edge belongs to $\Lambda$ and its orientation is opposite the orientation of $\Lambda$, and is 0 otherwise.

Definition 5 (Set of cycle and fundamental cycle vectors). The set of cycle vectors is $L=\left\{1_{\Lambda}, \quad \forall \Lambda \in L\left(\mathcal{G}_{d}\right)\right\}$. A set of fundamental cycle vectors $L_{f} \subset L$ is a subset of $L$ that constitutes a basis for $L$. The elements of $L_{f}$ are called fundamental cycle vectors.

Given a set of fundamental cycle vectors $L_{f}$, we let $L_{f}\left(\mathcal{G}_{d}\right)$ denote the associated fundamental cycles $L_{f}(\mathcal{G})=\{\Lambda \in$ $\left.L\left(\mathcal{G}_{d}\right) \mid 1_{\Lambda} \in L_{f}\right\}$.

Definition 6 (Cycle and fundamental cycle matrix). The cycle matrix $C$ of a directed graph $G_{d}$ is the $k \times|\mathcal{E}|$ matrix $C=$ $\left[1_{\Lambda_{1}}, \ldots, 1_{\Lambda_{k}}\right]^{\top}$ where $k$ is the dimension of L. The $r \times|\mathcal{E}|$ matrix $C_{f} \subset C$, with $r=\operatorname{dim}\left(L_{f}\right)$, such that each row represents a fundamental cycle vector in $L_{f}$, is called the fundamental cycle matrix

$$
C_{f}=\left[1_{\Lambda_{1}}, \ldots, 1_{\Lambda_{r}}\right]^{\top}, \forall 1_{\Lambda_{i}} \in L_{f}
$$

Note that $C_{f}$ is not unique since it depends on the choice of the fundamental cycles vectors, and it is a full rank matrix.

For more information on how to calculate fundamental sets of cycles one may refer to Welch (1966).

Let $N_{i}$ be the set of all the nodes $j$ such that $(i, j) \in \mathcal{E}_{d}$. Define $\mathbf{b}_{i} \in \mathbb{R}^{\left|N_{i}\right|}$, as the vector obtained from stacking all the pseudomeasurements $\widetilde{\beta}_{i j}$ calculated from solving (3), $\forall j \in N_{i}$. Now let $\mathbf{b}=\left[\mathbf{b}_{1}^{\top}, \cdots, \mathbf{b}_{N}^{\top}\right]^{\top}$, and $\Theta$ be the vector of the to be calculated estimates, where its $k$-th entry corresponds to the estimate of the $k$-th entry of $\mathbf{b}$.

We want to solve the estimation problem in a least square sense, hence we have

$$
\begin{array}{cl}
\underset{\Theta \in \mathbb{R}^{|\mathcal{E}|}}{\operatorname{minimize}} & \|\mathbf{b}-\Theta\|^{2} \\
\text { subject to } & C_{f}^{\top} \Theta=0
\end{array}
$$

Moreover, we recast the constraint as $\Theta \in \operatorname{ker}\left(C_{f}^{\top}\right)$. Assume $\operatorname{ker}\left(C_{f}^{\top}\right)$ has $\delta_{1}, \cdots, \delta_{m}$ as a basis so we have

$$
\Theta=\sum_{i=1}^{m} \alpha_{i} \delta_{i}
$$

And the optimization problem (7) transforms into

$$
\underset{\alpha_{1}, \cdots, \alpha_{m} \in \mathbb{R}}{\operatorname{minimize}}\left\|\mathbf{b}-\sum_{i=1}^{m} \alpha_{i} \delta_{i}\right\|^{2}
$$

which can be solved easily. The solution to (7) is a constrained maximum likelihood estimate under the adopted error assumptions. Furthermore, note that if the graph does not have any cycles (it is a tree), the solution to (7), will be the same as the solution obtained from solving the unconstrained equation (3) for all the edges in the network, in other words $\Theta=\mathbf{b}$.

\section{A DISCRETE-TIME DISTRIBUTED CLOCK SYNCHRONIZATION SCHEME}

In this section we propose a cyclicly-distributed method for solving the relative clock synchronization problem subject to the cycle constraints introduced in the previous section.

Recall we want to solve the following least squares problem

$$
\begin{array}{cl}
\underset{\Theta \in \mathbb{R}^{|\mathcal{E}|}}{\operatorname{minimize}} & \|\mathbf{b}-\Theta\|^{2} \\
\text { subject to } & C_{f}^{\top} \Theta=0
\end{array}
$$

where $\mathbf{b}=\left[\mathbf{b}_{1}^{\top}, \cdots, \mathbf{b}_{N}^{\top}\right]^{\top}$ and $\mathbf{b}_{i} \in \mathbb{R}^{\left|N_{i}\right|}$ is the vector obtained from stacking all the pseudo-measurements $\widetilde{\beta}_{i j}$ calculated from solving (3), $\forall j \in N_{i}$. Again, $\Theta$ is the estimated vector of clock biases where the $k$-th entry corresponds to the estimate of the $k$-th entry of $\mathbf{b}$.

Now we want to solve (10) in a cyclicly-distributed iterative fashion, in a similar way to Piovan et al. (2008). Consider the following iterative algorithm executed at the $i^{t h}$ sensor for the link $e=(i, j)$

$$
\theta_{e}(t+1)=\theta_{e}(t)-\kappa \sum_{\substack{\Lambda_{l} \\ \text { s.t. } e \in \Lambda_{l} \\ \Lambda_{l} \in L_{f}}} 1_{\Lambda_{l}}^{\top} \Theta(t)
$$

where $\theta_{e}(0)=\widetilde{\beta}_{i j}$ when $e=(i, j)$ and $\kappa>0$ is specified later. As $t \rightarrow \infty$ we find that $\theta_{e}(t)$ approaches the constrained least-squares estimate of $\beta_{i j}$ and is across the entire network this equates to solving (7) in a cyclicly-distributed fashion (the proof of this equivalence is given in the next theorem). We say this iterative algorithm is cyclicly-distributed because it is distributed in the sense that sensor $i$ only requires information from the set of sensors on the fundamental cycles in $L_{f}\left(\mathcal{G}_{d}\right)$ on which it is a node.

Theorem 2. If $0<\kappa<2 /\left(1+\lambda_{\max }(F)\right)$ where $F=C_{f} C_{f}^{\top}$ and $\lambda_{\max }(F)$ is the maximum eigenvalue of $F$, then the solution of the discrete-time system (11) converges exponentially fast with exponential convergence factor $\rho=(1-\kappa)^{2}$ to the set of times $\theta_{i j}$ such that $C_{f}^{\top} \Theta=0$. Moreover, (11) converges exponentially fast to the solution of (10).

Proof. Let $\operatorname{dim}\left(L_{f}\right)=r$. Given the fundamental cycle matrix $C_{f}$ and the associated cycles $\Lambda_{1}, \ldots, \Lambda_{r}$, define the cycle error vector $\epsilon$ at $\Theta$ by $\epsilon=\left[\begin{array}{lll}\epsilon_{\Lambda_{1}} & \ldots & \epsilon_{\Lambda_{r}}\end{array}\right]^{\top}$ where $\epsilon_{\Lambda_{i}}$ is defined by $1_{\Lambda_{i}} \cdot \Theta$ for all $i \in\{1, \ldots, r\}$. With this notation we have

$$
\Theta(t+1)=\Theta(t)-\kappa C_{f}^{\top} \epsilon(t)
$$

where $\mathbf{1}_{\epsilon, \Lambda_{l}}$ is equal to $\epsilon$ with all elements equated to zero except the entry $\epsilon_{\Lambda_{l}}$. From this we can write down an iterative equation for the cycle error vectors

$$
\begin{aligned}
& \epsilon(t+1)=\epsilon(t)-\kappa C_{f} C_{f}^{\top} \epsilon(t) \\
& \epsilon(t+1)=(I-\kappa F) \epsilon(t)
\end{aligned}
$$


where $F=C_{f} C_{f}^{\top}$. Consider the Lyapunov equation $v(\epsilon)=$ $\epsilon^{\top} \epsilon$ such that

$$
v(\epsilon(t+1))-v(\epsilon(t))=\epsilon^{\top}\left(A^{\top} A-I\right) \epsilon
$$

where $A=I-\kappa F$. We need to show that there exists a positive definite $Q$ such that $A^{\top} A-I<-Q$. Write $F=$ $U V U^{\top}$ where $U$ is orthogonal and $V$ is a diagonal matrix $V=\operatorname{diag}\left(\lambda_{1}, \ldots, \lambda_{r}\right)$ and $\lambda_{i}$ is the $i^{t h}$ eigenvalue of $F$. Pick $Q=\left(2 \kappa-\kappa^{2}\right) I$ and thus

$$
U(I-\kappa V)^{\top}(I-\kappa V) U^{\top}-I \leq-U\left(2 \kappa-\kappa^{2}\right) U^{\top}
$$

which is satisfied if and only if

$$
\left(1-\kappa \lambda_{i}\right)^{2}-1+2 \kappa-\kappa^{2}<0, \forall i \in\{1, \ldots, r\}
$$

or alternatively if $\kappa<2 /\left(1+\lambda_{\max }(F)\right)$ since $\lambda_{\min }(F) \geq 1$; see Arioli et al. (2006). Also, $I-Q=\rho I$ where $\rho=(1-\kappa)^{2}$.

Note that equation (13) is the orthogonal projection of the cycle errors onto the subspace spanned by the constraints which is exactly the solution to the constrained least-squares problem (10) or (7). This completes the proof.

The preceding theorem states that the cyclicly-distributed iterative algorithm converges exponentially fast to the desired leastsquares solution such that the cycle errors, i.e. the sum of the relative clock biases around the fundamental cycles, are zero.

We can find a particular network topology such that each network node requires only the bias estimates associated with the edges incident to its one-hop neighbours.

Proposition 1. If $\mathcal{G}$ is a triangulation graph, then $\exists L_{f}\left(\mathcal{G}_{d}\right)$ on the associated directed graph $\mathcal{G}_{d}$ such for any $\Lambda \in L_{f}$ it holds that $|\Lambda|=3$, i.e. each fundamental cycle has exactly 3 vertices.

Proof. From Euler's characteristic it follows that the number of faces of a triangulation is equal to $2-|\mathcal{V}|+\left|\mathcal{E}_{c}\right|$ or, excluding the outer face, the number of triangular faces is equal to $1-|\mathcal{V}|+$ $\left|\mathcal{E}_{c}\right|$. It follows from the MacLane planarity criterion that the boundaries of the faces, of a planar graph, form cycles whose edge sets form a basis for the cycle space.

\section{A CONTINUOUS-TIME DISTRIBUTED CLOCK SYNCHRONIZATION SCHEME}

We extend the discrete-time iterative algorithm outlined in the previous section to a continuous-time algorithm.

Consider the following iterative algorithm executed at the $i^{t h}$ sensor for the link $e=(i, j)$

$$
\dot{\theta}_{e}=-\kappa \sum_{\substack{\Lambda_{l} \\ \text { s.t. } e \in \Lambda_{l} \\ \Lambda_{l} \in L_{f}}} 1_{\Lambda_{l}}^{\top} \Theta
$$

where $\theta_{e}(0)=\widetilde{\beta}_{i j}$ when $e=(i, j)$ and $\kappa>0$. As $t \rightarrow \infty$ we find that $\theta_{e}(t)$ approaches the constrained least-squares estimate of $\beta_{i j}$ and is across the entire network this equates to solving (7) in a cyclicly-distributed fashion (the proof of this equivalence is given in the next theorem). Again, as in the previous section, we say this iterative algorithm is cycliclydistributed because it is distributed in the sense that sensor $i$ only requires information from the set of sensors on the fundamental cycles in $L_{f}\left(\mathcal{G}_{d}\right)$ on which it is a node.
Theorem 3. If $\kappa>0$ then the solution of the continuous-time system (17) converges exponentially fast to the set of times $\theta_{i j}$ such that $C_{f}^{\top} \Theta=0$. Moreover, (17) converges exponentially fast to the solution of (10).

Proof. Let $\operatorname{dim}\left(L_{f}\right)=r$. Given the fundamental cycle matrix $C_{f}$ and the associated cycles $\Lambda_{1}, \ldots, \Lambda_{r}$, define the cycle error vector $\epsilon$ at $\Theta$ by $\epsilon=\left[\begin{array}{lll}\epsilon_{\Lambda_{1}} & \ldots & \epsilon_{\Lambda_{r}}\end{array}\right]^{\top}$ where $\epsilon_{\Lambda_{i}}$ is defined by $1_{\Lambda_{i}} \cdot \Theta$ for all $i \in\{1, \ldots, r\}$. With this notation we have

$$
\dot{\Theta}=-\kappa C_{f}^{\top} \epsilon
$$

where $\mathbf{1}_{\epsilon, \Lambda_{l}}$ is equal to $\epsilon$ with all elements equated to zero except the entry $\epsilon_{\Lambda_{l}}$. From this we can write down an iterative equation for the cycle error vectors

$$
\dot{\epsilon}=-\kappa C_{f} C_{f}^{\top} \epsilon
$$

which is stable at the origin for any $\kappa>0$.

Note that equation (19) is the orthogonal projection of the cycle errors onto the subspace spanned by the constraints which is exactly the solution to the constrained least-squares problem (10) or (7). This completes the proof.

The preceding theorem states that the cyclicly-distributed iterative algorithm converges exponentially fast to the desired leastsquares solution such that the cycle errors, i.e. the sum of the relative clock biases around the fundamental cycles, are zero. However, an advantage of the continuous-time formulation is that there is a simple algorithm addition, inspired by the results presented in Cao and Ren (2010); Cao et al. (2010), one can make to achieve a considerable speed-up in convergence. Specifically, one can achieve finite-time convergence if one is willing to assume that each node in the network knows the the set of fundamental cycles and more specifically the fundamental cycle matrix $C_{f}$. Such knowledge is obtainable in practice from the topology of the network. Consider the following iterative algorithm executed for the entire network

$$
\dot{\Theta}=-\kappa C_{f}^{\top} \epsilon(t)-\zeta C_{f}^{\#} \operatorname{sgn}\left(C_{f} C_{f}^{\top} \epsilon(t)\right)
$$

where $\Theta(0)=\mathbf{b}$ and $\kappa, \zeta>0$ and $C_{f}^{\#}$ is the generalized inverse. Note that for a particular network and in particular a specifed $C_{f}$ we can extract a differential equation for $\dot{\theta}_{e}$ as before.

Theorem 4. If $\kappa, \zeta>0$ then the solution of the continuoustime system (20) and, in particular, for a specified $C_{f}$ the continuous-time equation for $\dot{\theta}_{e}$ converges in finite-time period to the set of times $\theta_{i j}$ such that $C_{f}^{\top} \Theta=0$. Moreover, (20) converges in a finite-time period to the solution of (10).

Proof. Let $\operatorname{dim}\left(L_{f}\right)=r$. Given the fundamental cycle matrix $C_{f}$ and the associated cycles $\Lambda_{1}, \ldots, \Lambda_{r}$, define the cycle error vector $\epsilon$ at $\Theta$ by $\epsilon=\left[\begin{array}{lll}\epsilon_{\Lambda_{1}} & \ldots & \epsilon_{\Lambda_{r}}\end{array}\right]^{\top}$ where $\epsilon_{\Lambda_{i}}$ is defined by $1_{\Lambda_{i}} \cdot \Theta$ for all $i \in\{1, \ldots, r\}$. We can write down an iterative equation for the cycle error vectors

$$
\dot{\epsilon}=-\kappa C_{f} C_{f}^{\top} \epsilon-\zeta \operatorname{sgn}\left(C_{f} C_{f}^{\top} \epsilon\right)
$$

Pick $v=\frac{1}{2} \epsilon^{\top} F \epsilon$ where $F=C_{f} C_{f}^{\top}$. It follows that 


$$
\begin{aligned}
\dot{v} & =\epsilon^{\top} F(-\kappa F \epsilon-\zeta \operatorname{sgn}(F \epsilon)) \\
& \leq-\kappa \epsilon^{\top} F^{2} \epsilon-\zeta\|F \epsilon\|_{1} \\
& \leq-\zeta\|F \epsilon\| \\
& \leq-\zeta \sqrt{\lambda_{\min }^{2}(F)}\|\epsilon\| \\
& \leq-\zeta \frac{2 \lambda_{\min }(F)}{\lambda_{\max }(F)} \sqrt{v}
\end{aligned}
$$

Then it follows that

$$
2 \sqrt{v} \leq 2 \sqrt{v(0)}-\zeta \frac{2 \lambda_{\min }(F)}{\lambda_{\max }(F)} t
$$

and thus $v(t)=0$ for all $t \geq T$ where

$$
T=\frac{\sqrt{\lambda_{\max }(F) \epsilon^{\top}(0) F \epsilon(0)}}{\zeta \lambda_{\min }(F)}
$$

and $T<\infty$.

Note that equation (21) is the orthogonal projection of the cycle errors onto the subspace spanned by the constraints which is exactly the solution to the constrained least-squares problem (10) or (7). The addition of the $\zeta \operatorname{sgn}\left(C_{f} C_{f}^{\top} \epsilon\right)$ does not change the direction of motion for $\epsilon$ but rather it changes only the magnitude of the motion velocity. This completes the proof.

In the next section we present an example in which we compare the result obtained from solving the constrained optimization introduced here and the result obtained from solving the unconstrained equations of type (3).

\section{ILLUSTRATIVE EXAMPLE}

Consider a network of 100 nodes (An example of such network is depicted in Fig. 1(a)). Relative clock bias estimates are calculated at node 1 for different levels of Gaussian measurement noise (node 1 is assumed to have access to all the measurements in the network.). The mean-square error in the clock bias estimate is computed over 1000 runs. The position of the nodes are randomly generated during each run according to a uniform distribution and the topology of the network is the standard random geometric graph. The mean-square error of the estimates for both unconstrained and constrained solutions are compared in Fig. 1(b).

\section{CONCLUSION}

In this paper we first formalized in what type of graph structure can one compute all the relative clock biases between each pair of nodes. We then reviewed a centralized method based on solving a convex constrained optimization problem to estimate relative clock biases in a (sensor) network. We the introduced a number of distributed mechanisms for solving the stated constrained optimization algorithm. We provided a discretetime solution that is guaranteed to converge to the optimal constrained linear least-squares solution with an exponential speed. Then we provided a similar algorithm for achieving the same solution in continuous-time. Both the discrete-time algorithm and the continuous-time algorithm are distributed in that each node in the network requires very little information from its neighbours in the network.

Finally, we provide an algorithm that achieves a finite-time convergence to the desired solution in a distributed manner.

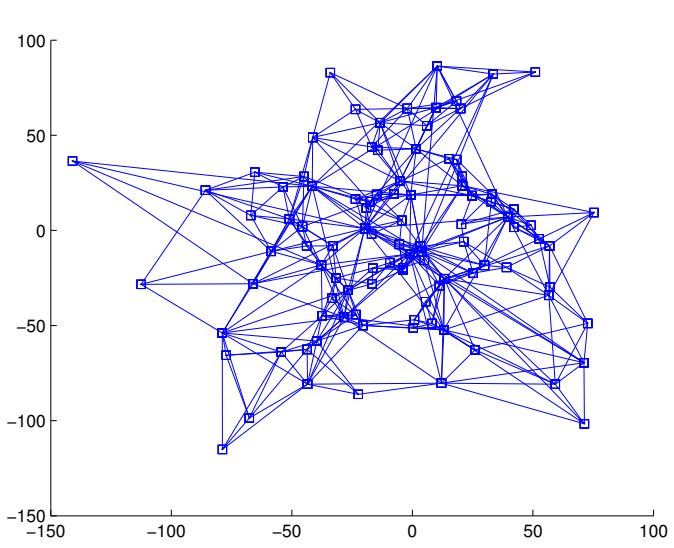

(a) An example of a network with 100 nodes that is considered here.

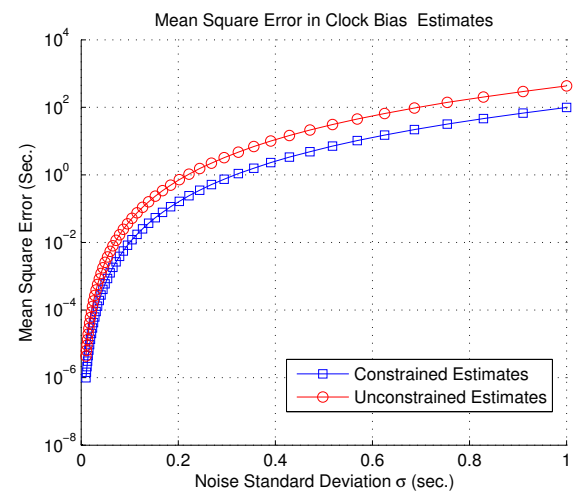

(b) The mean square error of clock bias estimates for different noise levels.

This algorithm requires that each node in the network have access to knowledge concerning the network topology and in particular certain kinds of network cycles.

The constrained optimization methods will in general outperform the simple unconstrained method already available in the literature. Simulation results are presented to back this claim.

\section{REFERENCES}

Arioli, M., Maryska, J., Rozloznik, M., and Tuma, M. (2006). Dual variable methods for mixed-hybrid finite element approximation of the potential fluid flow problem in porous media. Electronic Transactions on Numerical Analysis, 22, 17-40.

Bachrach, J. and Taylor, C. (2005). Handbook of sensor networks: algorithms and architectures. Wiley.

Barahona, M. and Pecora, L. (2002). Synchronization in smallworld systems. Physical Review Letters, 89, 054101-(1-4).

Barooah, P. and Hespanha, J. (2005). Distributed Estimation from Relative Measurements in Sensor Networks. In Proceedings of the 3rd International Conference on Intelligent Sensing and Information Processing, 226-231.

Beck, A., Stoica, P., and Li, J. (2008). Exact and approximate solutions of source localization problems. IEEE Transactions on Signal Processing, 56, 1770-1778.

Biswas, P., Lian, T.C., Wang, T.C., and Ye, Y. (2006). Semidefinite programming based algorithms for sensor network localization. ACM Transaction on Sensor Networks, 2(2), 188220 . 
Biswas, P. and Ye, Y. (2004). Semidefinite programming for ad hoc wireless sensor network localization. In ACM IPSN, 46-54.

Bolognani, S., Carli, R., and Zampieri, S. (2009). A PI consensus controller with gossip communication for clock synchronization in wireless sensors networks. In Proceedings of the 1st IFAC Workshop on Estimation and Control of Networked Systems.

Bruck, J., Gao, J., and Jiang, A.A. (2009). Localization and routing in sensor networks by local angle information. $A C M$ Transactions on Sensor Networks (TOSN), 5(1), 7.

Cao, Y. and Ren, W. (2010). Distributed coordinated tracking via a variable structure approach - part i: Consensus tracking. In Proceedings of American Control Conference.

Cao, Y., Ren, W., and Meng, Z. (2010). Decentralized finitetime sliding mode estimators with applications to formation tracking. In Proc. of the 2010 American control Conference, 4610-4615.

Carter, M.W., Jin, H.H., Saunders, M.A., and Ye, Y. (2006). An adaptive subproblem algorithm for scalable wireless sensor network localization. SIAM Journal on Optimization, 17(4), 1102-1128.

Costa, J.A., Patwari, N., and Hero III, A.O. (2006). Distributed weighted-multidimensional scaling for node localization in sensor networks. ACM Transactions on Sensor Networks (TOSN), 2(1), 39-64.

Cristian, F. (1989). Probabilistic clock synchronization. Distributed Computing, 3(3), 146-158.

Ding, Y., Krislock, N., Qian, J., and Wolkowicz, H. (2010). Sensor network localization, Euclidean distance matrix completions, and graph realization. Optimization and Engineering, 11(1), 45-66.

Doherty, L., Piste, K.S.J., and ElGhaoui, L. (2001). Convex position estimation in wireless sensor networks. In IEEE INFOCOM, volume 3, 1655-1663.

Elson, J., Girod, L., and Estrin, D. (2002). Fine-grained network time synchronization using reference broadcasts. In Proceedings of the 5th Symposium on Operating Systems Design and Implementation.

Freris, N., Borkar, V., and Kumar, P.R. (2009). A model-based approach to clock synchronization. In Proceedings of the 46th IEEE Conference on Decision and Control, 5744-5749. Shanghai, China.

Freris, N. and Kumar, P.R. (2007). Fundamental limits on synchronization of affine clocks in networks. In Proceedings of the 46th IEEE Conference on Decision and Control, 921926. New Orleans, USA.

Ganeriwal, S., Kumar, R., and Srivastava, M. (2003). Timingsync protocol for sensor networks. In Proceedings of the 1st International Conference on Embedded Networked Sensor Systems.

Giridhar, A. and Kumar, P.R. (2006). Distributed clock synchronization over wireless networks: Algorithms and analysis. In Proceedings of the 46th IEEE Conference on Decision and Control, 4915-4920. San Diego, CA, USA.

Lederer, S., Wang, Y., and Gao, J. (2009). Connectivity-based localization of large-scale sensor networks with complex shape. ACM Transaction on Sensor Networks, 5(4), 1-32.

Maroti, M., Kusy, B., Simon, G., and Ledeczi, A. (2004). The flooding time synchronization protocol. In Proceedings of the 2nd International Conference on Embedded Networked Sensor Systems.
Moore, D., Leonard, J., Rus, D., and Teller, S. (2004). Robust distributed network localization with noisy range measurements. In Proc. of the 2nd International Conference on Embedded Networked Sensor Systems, 50-61. Baltimore, USA.

Nie, J. (2009). Sum of squares method for sensor network localization. Computational Optimization and Applications, 43(2), 151-179.

Piovan, G., Shames, I., Fidan, B., Bullo, F., and Anderson, B.D.O. (2008). On frame and orientation localization for relative sensing networks. In Proceedings of 47th IEEE Conference on Decision and Control, 2326-2331. Cancun, Mexico.

Sallai, J., Kusy, B., Ledeczi, A., and Dutta, P. (2006). On the scalability of routing integrated time synchronization. In Proceedings of the 3rd European Workshop on Wireless Sensor Networks.

Schenato, L. and Gamba, G. (2007). A distributed consensus protocol for clock synchronization in wireless sensor network. In Proceedings of the 46th IEEE Conference on Decision and Control.

Shames, I., Anderson, B., and Fidan, B. (2009a). On the use of convex optimization in sensor network localization and synchronization. In Proceedings of the 1st IFAC Workshop on Estimation and Control of Networked Systems.

Shames, I., Bibalan, P.T., Fidan, B., and Anderson, B.D.O. (2009b). Polynomial methods in noisy network localization. In Proc. of the 17th Mediterranean Conference on Control and Automation, 1307-1312. Thessaloniki, Greece.

Shames, I. and Bishop, A. (2010). Relative clock synchronization in wireless networks. IEEE Communication Letters, 14(4), 348-350.

Shang, Y., Ruml, W., Zhang, Y., and Fromherz, M.P.J. (2003). Localization from mere connectivity. In Proc. of the 4th ACM international symposium on Mobile ad hoc networking \& computing, 201-212. Annapolis, Maryland, USA.

Simeone, O. and Spagnolini, U. (2007). Distributed time synchronization in wireless sensor networks with coupled discrete-time oscillators. EURASIP Journal on Wireless Communications and Networking, 57054.

Solis, R., Borkar, V., and Kumar, P.R. (2006). A new distributed time synchronization protocol for multihop wireless networks. In Proceedings of the 46th IEEE Conference on Decision and Control, 2734-2739. San Diego, CA, USA.

Sommer, P. and Wattenhofer, R. (2009). Gradient clock synchronization in wireless sensor networks. In Proceedings of the 2009 International Conference on Information Processing in Sensor Networks.

Sundararaman, B., Buy, U., and Kshemkalyani, A. (2005). Clock synchronization for wireless sensor networks: A survey. Ad Hoc Networks, 3(3), 281-323.

Wang, X.F. and Chen, G. (2002). Synchronization in scalefree dynamical networks: Robustness and fragility. IEEE Transactions on Circuits and Systems-I, 49, 54-62.

Welch, J.T. (1966). A mechanical analysis of the cyclic structure of undirected linear graphs. J. ACM, 13, 205-210.

Xiong, G. and Kishore, S. (2008). Convergence properties of a second order consensus-based time synchronization algorithm for wireless sensor networks. In Proceedings of the 2008 IEEE Global Communications Conference. 